\title{
Prefixation with clip-anchored endoloop: a novel method facilitating endoscopic resection of a giant duodenal polyp with thick stalk
}

Although pretreatment of the stalk is a standard method to prevent bleeding for endoscopic removal of large pedunculated lesions [1], adequate stalk ligation is still challenging in some difficult cases [2]. In this study, we report a prefixation technique to improve endoscopic treatment of a giant duodenal polyp by reliably ligating the stalk with a clip-anchored endoloop ( $>$ Video 1).

A symptomatic 49-year-old woman was diagnosed with a giant pedunculated polyp $(5.0 \mathrm{~cm})$ extending from the duodenal bulb to the descending duodenum under esophagogastroduodenoscopy ( $\triangleright$ Fig. 1 a). Because the mass almost occluded the duodenal lumen, we decided to remove it endoscopically based on its benign appearance. Because the enormous mass spared little space and the long, thick stalk $(1.5 \mathrm{~cm})$ moved freely in the small lumen, sufficient mass could not be ensnared with an endoloop ( Fig. 1 b) [3] even with the aid of grasping forceps ( $>$ Fig. 1 c) [2]. The endoloop was then preloaded in the working channel of the endoscope and extended out to link with a clip ( $\triangleright$ Fig. 2 a). Because the endoloop was pre-fixed to the base of the mass with the clip ( $\triangleright$ Fig. 2 b), it perfectly trapped the mass and ligated its thick stalk by means of the clip-created fulcrum ( $\triangleright$ Fig. $2 \mathbf{c}$ ). With the stalk cut off by a hook knife ( $>$ Fig. 2 d), a neat base along with the clip-anchored

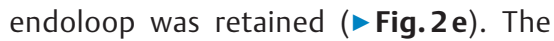
resected specimen was extracted and measured (> Fig. 2f). The histology diagnosed the large polyp as a Brunner's gland hamartoma without malignancy ( Fig.3) [4].

Because the prefixation technique using a clip-anchored endoloop could reliably ligate the thick stalk pre-resection, precisely set the resecting edge to achieve

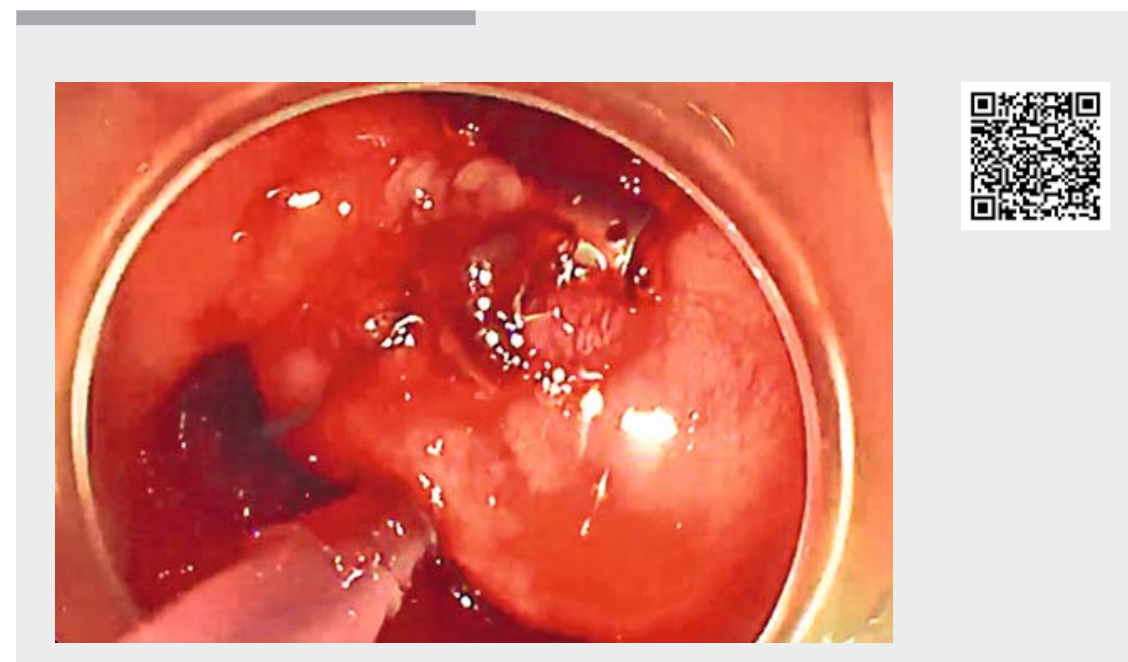

$\checkmark$ Video 1 Pre-fix technique with clip-anchored endoloop facilitating successful endoscopic resection of a giant duodenal pedunculated polyp.
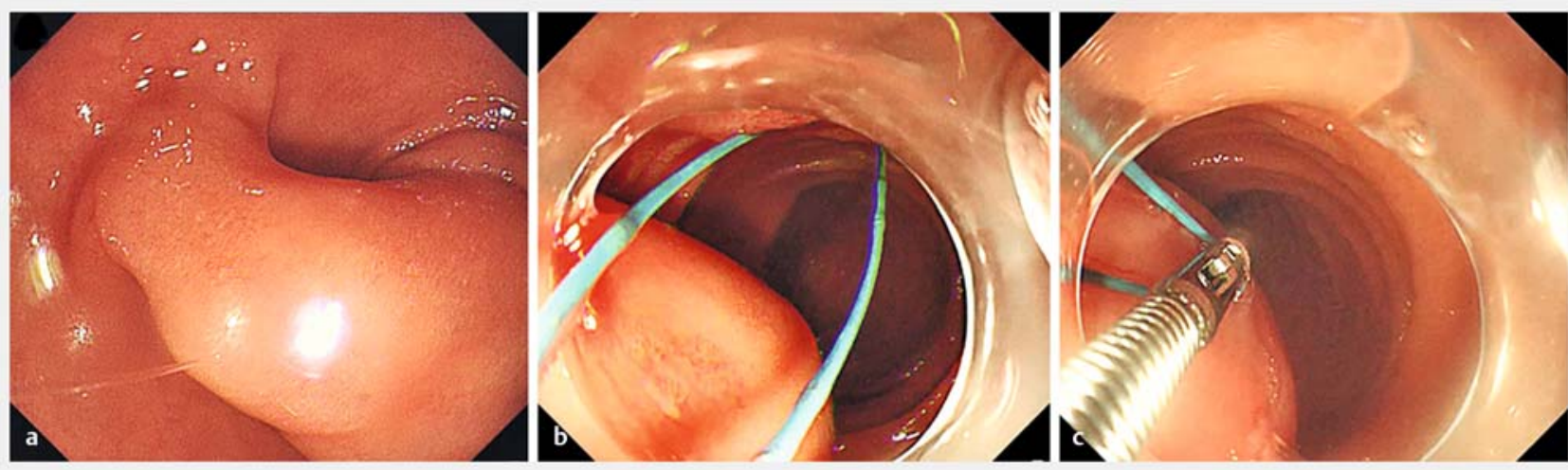

- Fig. 1 Failed endoscopic treatment of a giant duodenal pedunculated polyp using conventional methods. a Esophagogastroduodenoscopy presents a huge pedunculated mass occupying the lumen of the duodenal bulb and extending to the descending part of the duodenum. b Ligation of the thick stalk of the mass by an endoloop was attempted but failed. c The grasping forceps were unsuccessful in helping the endoloop ensnare the large mass. 

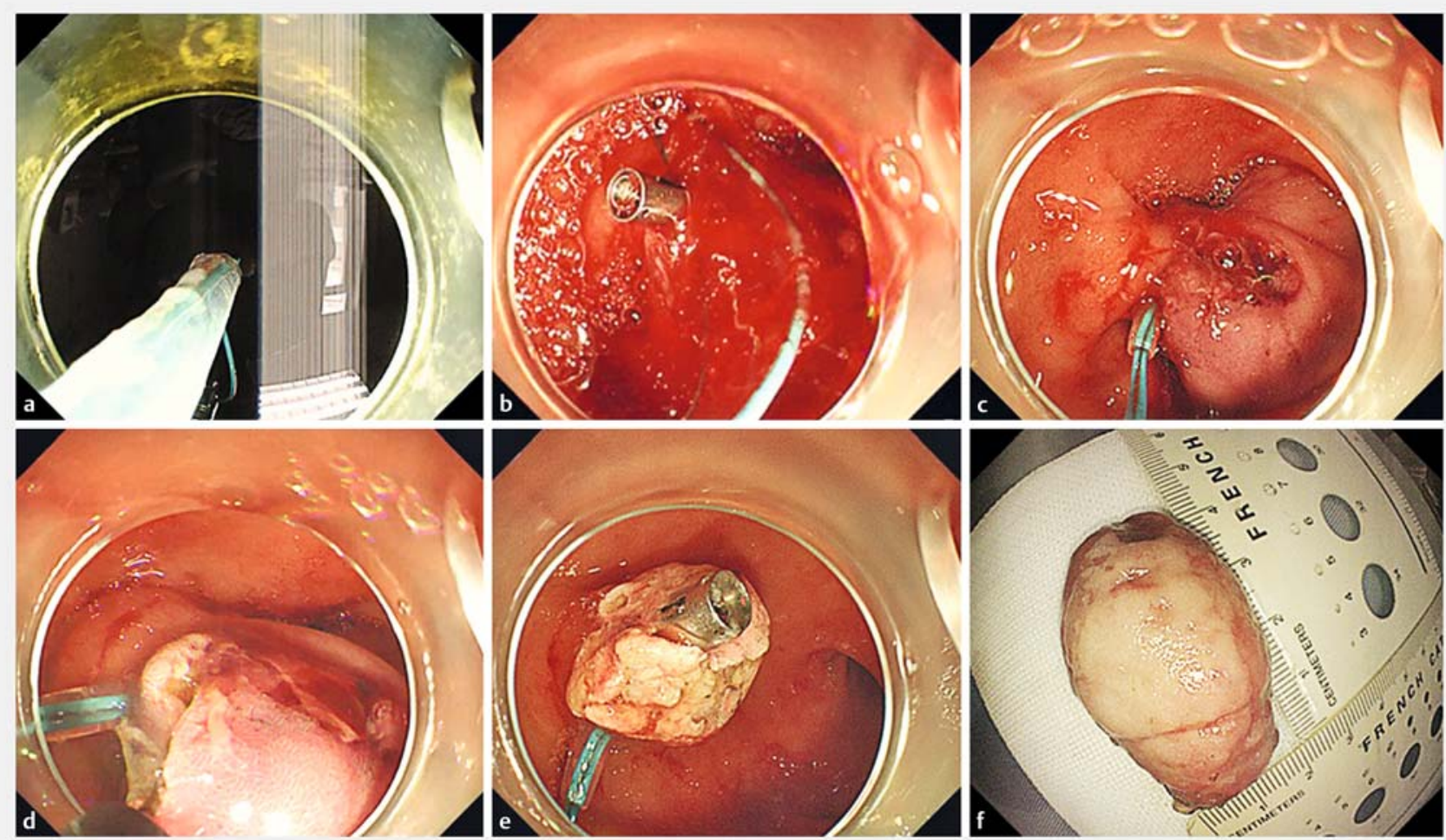

- Fig. 2 Pre-fix technique with clip-anchored endoloop facilitates successful endoscopic resection of a giant duodenal pedunculated polyp. a An endoloop was extended out of the working channel of the endoscope and then equipped with a clip. $\mathbf{b}$ The endoloop was fixed to the root of the giant mass by the clip. $\mathbf{c}$ The thick stalk of the mass was completely ligated by the endoloop. $\mathbf{d}$ A hook knife was introduced to resect the giant mass. e A neat base after resection was well-fastened by the clip-anchored endoloop. $\mathbf{f}$ The resected specimen measured $5.0 \times 2.5 \mathrm{~cm}$.

convenient removal of the whole lesion and solid preservation of the normal tissue, and dependably avoid endoloop slippage-related bleeding post-resection, it may serve as a standard treatment for all pedunculated lesions.

\section{Endoscopy_UCTN_Code_TTT_1AO_2AG}

\section{Competing interests}

The authors declare that they have no conflict of interest.
The authors

Xiao Hu1,2, Xu-dan Yang ${ }^{2,3}$, Ying-hui Zhang ${ }^{1,2}$, Cheng-hong $\mathrm{Li}^{1}{ }^{1,2}$, Sheng-xi Han ${ }^{1,2}$, Wei-hui Liu $^{1,2}$

1 Department of Gastroenterology and Hepatology, Sichuan Academy of Medical Sciences \& Sichuan Provincial People's Hospital, Chengdu, Sichuan Province, China

2 School of Medicine, University of Electronic Science and Technology of China, Chengdu, Sichuan Province, China

3 Department of Pathology, Sichuan Academy of Medical Sciences \& Sichuan Provincial People's Hospital, Chengdu, Sichuan Province, China
Corresponding author

\section{Wei-hui Liu, MD}

Department of Gastroenterology and Hepatology, Sichuan Academy of Medical Sciences \& Sichuan Provincial People's Hospital, Chengdu, Sichuan Province, 610072, China

Fax: +86-288-657-1251

audiliu12@163.com 


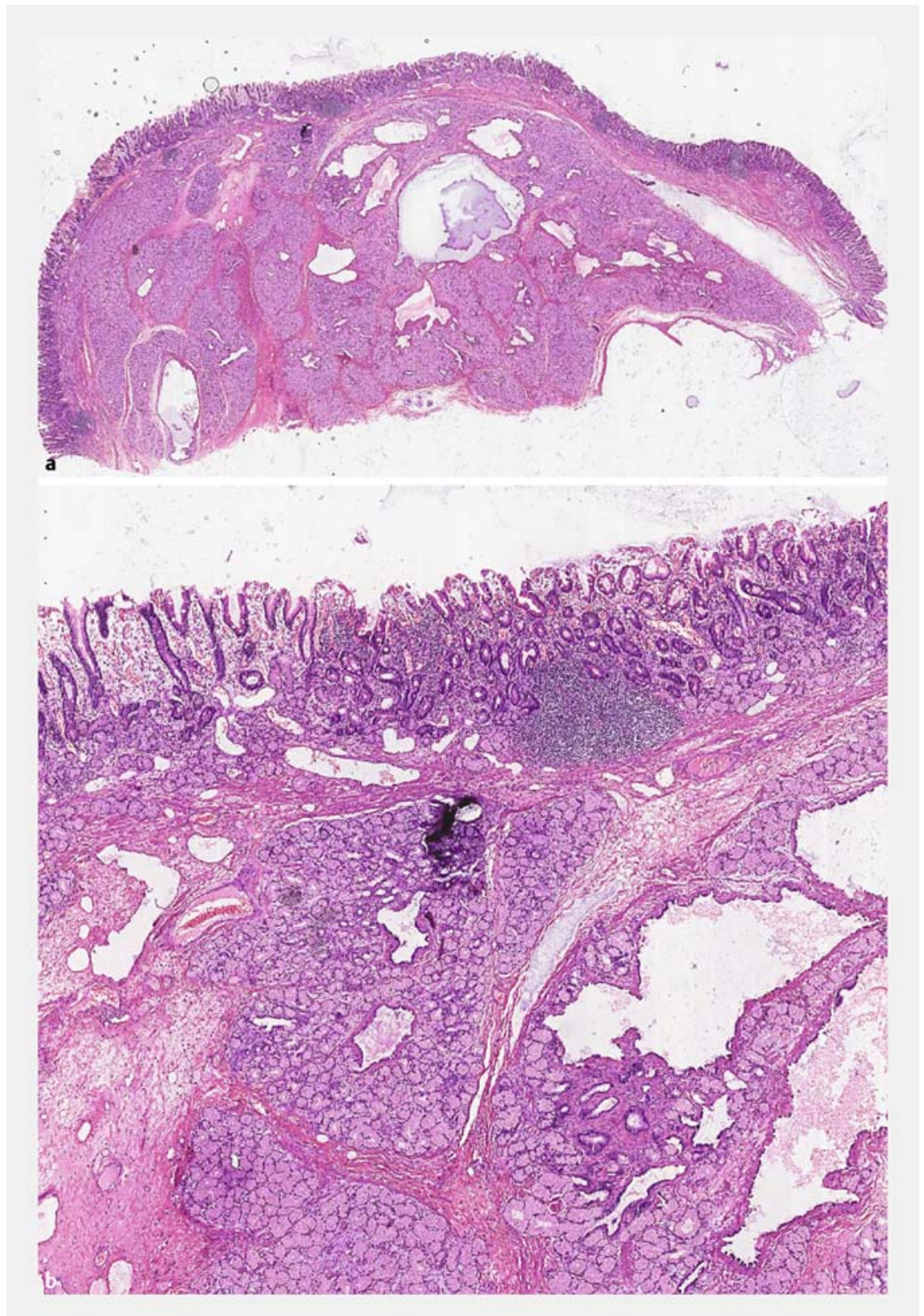

- Fig. 3 The histological appearance of the resected specimen showing submucosal proliferation of Brunner's glands with smooth muscle bundles consistent with Brunner's gland hamartoma. a Hematoxylin and eosin (H\&E) staining, original magnification $\times 10$. b H\&E staining, original magnification $\times 40$.
[1] Ferlitsch M, Moss A, Hassan C et al. Colorectal polypectomy and endoscopic mucosal resection (EMR): European Society of Gastrointestinal Endoscopy (ESGE) Clinical Guideline. Endoscopy 2017; 49: 270-297

[2] Curcio G, Ligresti D, Granata A et al. Grasping forceps assisted endoloop/snare polypectomy of a giant duodenal hamartoma. Therap Adv Gastroenterol 2015; 8: 313-315

[3] Huang CH, Perng CL, Yang YH et al. Endoscopic removal of a huge duodenal Brunner's gland adenoma: a new technique. Gastrointestinal endoscopy 1999; 50: 868869

[4] Sudo G, Kobayashi T, Nakase H. Brunner's gland hamartoma arising rrom the pyloric ring. Clin Gastroenterol Hepatol 2019; 17: e155

\section{Bibliography}

Endoscopy 2021; 53: E87-E89

DOI 10.1055/a-1198-4200

ISSN 0013-726X

published online 17.7.2020

(c) 2020. Thieme. All rights reserved.

Georg Thieme Verlag KG, Rüdigerstraße 14, 70469 Stuttgart, Germany

\section{ENDOSCOPY E-VIDEOS}

https://eref.thieme.de/e-videos

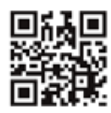
Endoscopy E-Videos is a free access online section, reporting on interesting cases and new techniques in gastroenterological endoscopy. All papers include a high quality video and all contributions are freely accessible online.

This section has its own submission website at https://mc.manuscriptcentral.com/e-videos 